\title{
ACOUSTIC WAVE TRANSMISSION ON HOMOGENIZED PERFORATED PLATE
}

\begin{abstract}
E. Rohan*, V. Lukeš ${ }^{* *}$
Abstract: We consider problems of the acoustic wave propagation in an inviscid fluid through a rigid periodically perforated plate. Using the homogenization method the wave propagation in a layer containing the plate is replaced by transmission conditions which couple the acoustic field on both sides of the plate represented by a flat interface. The interface impedance depends of the shape of the perforation. For suitable shapes of the holes, there is a coupling between transverse and surface acoustic waves propagating along the panel. The derived model leads to a significant savings in the computational cost of the numerical solutions.
\end{abstract}

Keywords: acoustic transmission, homogenization, acoustic impedance, perforated plate

\section{Introduction}

In this work we present a reduced model of the acoustic transmission on perforated plates. We pursue the approach proposed in Rohan and Lukeš (2010); therein, the homogenization of the Helmholtz equation in a layer perforated by rigid obstacles was treated to derive an effective model of the acoustic wave propagation in the so-called transmission layer. Before, in Bonnet-Bendhia et.al. (2005), only very thin perforated rigid sheets were considered. As an alternative to the approach employed in Marigo and Maurel (2016), we derived non-local transmission conditions which take into account the perforation geometry in a rigorous way. The new achievement reported in this article concerns with an improved version of these interface conditions which, besides the jump in the acoustic potential, also involve mean transverse acoustic momentum and its jump. The present approach can be adapted for modelling vibro-acoustic transmission on perforated compliant panels, as considered in Rohan and Lukeš (2013) in the context of the optimal perforation design problem.

We consider acoustic waves in an inviscid fluid characterized by the sound speed $c$, propagating with a frequency $\omega$ in a duct containing a perforated rigid plate. The homogenization method is applied to the Helmholtz equation governing the acoustic potential distributed in a fictitious layer $\Omega_{\delta} \subset \Omega^{G}$ in which the perforated plate is embedded; the size of the perforation is $\approx \varepsilon$. The asymptotic analysis is performed for the thickness $\delta=\varkappa \varepsilon \rightarrow 0$ with a fixed $\varkappa>0$, so that also the size of the holes in the plate tends to zero. This yields transmission conditions applied on the interface $\Gamma_{0}$ associated with the mid-plane of the layer $\Omega_{\delta}$. These conditions allow us to describe waves propagating in two domains $\Omega^{+}$and $\Omega^{-}$separated by the homogenized perforated plate represented by $\Gamma_{0}=\partial \Omega^{+} \cap \partial \Omega^{-}$. The solutions of the global problem in domain $\Omega^{G}=\Omega^{+} \cup \Omega^{-} \cup \Gamma_{0}$ are decomposed into a "background" field $P^{0}$ which disregards the perforated interface, cf. e.g. Bonnet-Bendhia et.al. (2005), and the first-order correction $\delta_{0} P^{1}$ which is computed using the interface conditions, whereby finite thickness $\delta_{0}=\varkappa \varepsilon_{0}>0$ of the layer is considered.

\section{Problem formulation and decomposition}

In this section, we introduce the problem of acoustic waves in a domain $\Omega^{G}$ with embedded perforated rigid plate $\Sigma^{\varepsilon}$. We consider a fictitious transmission layer $\Omega_{\delta}$ with a thickness $\delta>0$, such that $\Sigma^{\varepsilon} \subset \Omega_{\delta}$. The plate thickness is $h^{\varepsilon}=\varepsilon \bar{h}<\delta$, while the layer thickness $\delta=\varkappa \varepsilon$ for a given fixed $\varkappa>0$. Given

Prof. Dr. Ing. Eduard Rohan, DSc.: NTIS New Technologies for Information Society, Department of mechanics, Faculty of Applied Sciences, University of West Bohemia, Univerzitní 8, 30614, Pilsen, CZ, e-mail: rohan@kme.zcu.cz

** Ing. Vladimír Lukeš, Ph.D.: NTIS New Technologies for Information Society, Department of mechanics Faculty of Applied Sciences, University of West Bohemia in Pilsen, Univerzitní 22, 30614 Plzeň; CZ, e-mail: vlukes@kme.zcu.cz 


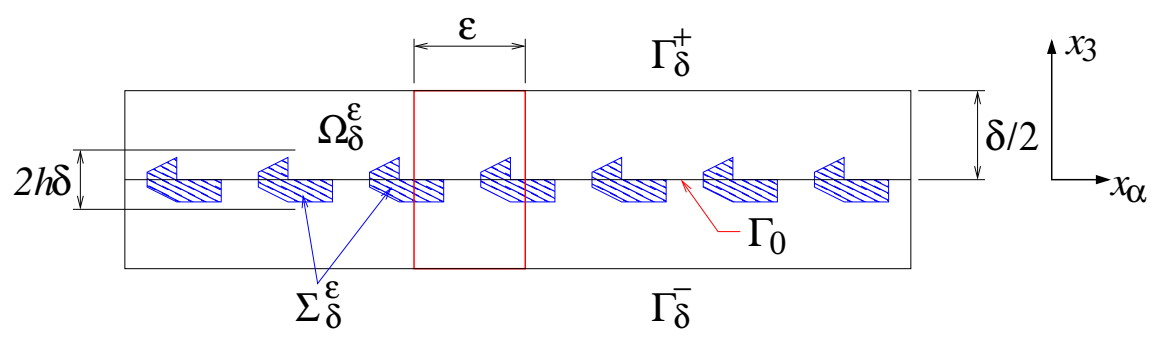

Fig. 1: Scheme of the transmission layer with periodically distribute obstacles; The layer thickness $\delta=\varkappa \varepsilon$, the obstacles (or the perforated plate) are embedded in layer of the thickness $h^{\varepsilon}=\varepsilon h$.

a bounded 2D manifold $\Gamma_{0} \subset\left\{x \in \Omega^{G} \mid x_{3}=0\right\}$ representing the plate mid-plane, we introduce $\Omega_{\delta}=$ $\left.\Gamma_{0} \times\right]-\delta / 2, \delta / 2\left[\subset \Omega^{G}\right.$, an open domain representing the transmission layer. This enables to decompose $\Omega^{G}$ into three non-overlapping parts, as follows: $\Omega^{G}=\Omega_{\delta} \cup \Omega_{\delta}^{+} \cup \Omega_{\delta}^{-}$. Thus, the transmission layer is bounded by $\partial \Omega_{\delta}$ which splits into three parts:

$$
\left.\partial \Omega_{\delta}=\Gamma_{\delta}^{+} \cup \Gamma_{\delta}^{-} \cup \partial_{\mathrm{ext}} \Omega_{\delta}, \quad \Gamma_{\delta}^{ \pm}=\Gamma_{0} \pm \frac{\delta}{2} \overrightarrow{e_{3}}, \quad \partial_{\text {ext }} \Omega_{\delta}=\partial \Gamma_{0} \times\right]-\delta / 2, \delta / 2[,
$$

where $\delta>0$ is the layer thickness and $\overrightarrow{e_{3}}=(0,0,1)$, see Fig. 1 . In the context of the transmission layer definition, we consider the plate as a $3 \mathrm{D}$ domain $\Sigma^{\varepsilon}$ generated using a representative structure as a periodic lattice.

The global acoustic problem can be split into two parts: 1) the out-of-layer problem imposed in subdomains $\Omega_{\delta}^{+}$and $\Omega_{\delta}^{-}$governs the acoustic potential $P^{\delta} ; 2$ ) the problem imposed in the layer, where $p^{\varepsilon}$ is the acoustic potential in the fluid part $\Omega_{\delta}^{* \varepsilon}=\Omega_{\delta} \backslash \Sigma^{\varepsilon}$. Further, by $\mathrm{i} \omega g^{\varepsilon \pm}$ we denote the acoustic fluid velocity projected into the normal of the interfaces $\Gamma_{\delta}^{ \pm}$. The following subproblems are considered:

1. Given $\hat{p}^{\varepsilon}$ on $\Gamma_{\delta}^{ \pm}$, find $P^{\delta}$ defined in $\Omega_{\delta}^{G}=\Omega_{\delta}^{+} \cup \Omega_{\delta}^{-}$, such that

$$
c^{2} \nabla^{2} P^{\delta}+\omega^{2} P^{\delta}=0 \quad \text { in } \Omega_{\delta}^{+} \cup \Omega_{\delta}^{-}, \quad P^{\delta}=\hat{p}^{\varepsilon} \quad \text { on } \Gamma_{\delta}^{ \pm},
$$

supplemented by boundary conditions $r i \omega c P^{\delta}+c^{2} \frac{\partial P^{\delta}}{\partial n}=s 2 \mathrm{i} \omega c \bar{p}$ imposed on the "external" boundary $\partial_{\text {ext }} \Omega_{\delta}^{G}=\partial \Omega^{G} \cap\left(\partial \Omega_{\delta}^{+} \cup \partial \Omega_{\delta}^{-}\right)$, where $r$ and $s$ are constants attaining values 0 , or 1 , whereas $\bar{p}$ is the amplitude of an incident wave.

2. Given $g^{\varepsilon \pm}$ on $\Gamma_{\delta}^{ \pm}$, find $p^{\varepsilon}$ in $\Omega_{\delta}^{* \varepsilon}$ and $\boldsymbol{u}^{\varepsilon}$ in $\Sigma^{\varepsilon}$, such that

$$
c^{2} \nabla^{2} p^{\varepsilon}+\omega^{2} p^{\varepsilon}=0 \quad \text { in } \Omega_{\delta}^{* \varepsilon}, \quad \frac{\partial p^{\varepsilon}}{\partial n}=-\mathrm{i} \omega g^{\varepsilon \pm} \quad \text { on } \Gamma_{\delta}^{ \pm}, \quad \frac{\partial p^{\varepsilon}}{\partial n}=0, \quad \text { on } \partial \Omega_{\delta}^{* \varepsilon} \cap \Omega_{\delta} .
$$

The two subproblems are coupled by the following conditions imposed on $\Gamma_{\delta}^{ \pm}$,

$$
\mathrm{i} \omega g^{\varepsilon \pm}=\frac{\partial P^{\delta}}{\partial n^{ \pm}} \quad \text { and } p^{\varepsilon}=\hat{p}^{\varepsilon}=P^{\delta}
$$

where $n^{ \pm}$refers to normals $\boldsymbol{n}^{ \pm}$outer to domains $\Omega_{\delta}^{ \pm}$.

\section{Homogenized transmission conditions and the global problem}

In Rohan and Lukeš (2010), using the asymptotic analysis of problem (3) for $\varepsilon \rightarrow 0$, we derived the limit problem in the homogenized layer represented by $\Gamma_{0}$. The limit acoustic potential $p^{0} \in H^{1}\left(\Gamma_{0}\right)$ solves the limit Helmholtz equation,

$$
\int_{\Gamma_{0}}\left(\boldsymbol{A} \bar{\nabla}_{x} p^{0}\right) \cdot \bar{\nabla}_{x} q^{0}-\frac{\omega^{2}}{c^{2}} \int_{\Gamma_{0}} p^{0} q^{0}+\mathrm{i} \omega \int_{\Gamma_{0}} \bar{\nabla}_{x} q^{0} \cdot \boldsymbol{B} \frac{\partial P^{0}}{\partial n}=-\mathrm{i} \omega \int_{\Gamma_{0}} q^{0}\left[\bar{g}^{1 \pm}\right]_{-}^{+}, \quad \forall q^{0} \in H^{1}\left(\Gamma_{0}\right)
$$

where $\bar{\nabla}_{x}=\left(\partial_{\alpha}\right)$ is the "in-plane" gradient, $\boldsymbol{A}=\left(A_{\alpha \beta}\right)$ is the acoustic velocity anisotropy and $\boldsymbol{B}=\left(B_{\alpha}\right)$ is a coupling vector; both $\boldsymbol{A}$ and $\boldsymbol{B}$ depend on the characteristic responses of the local "cell problems" 
which depend on the perforation geometry. Functions $g^{0}$ and $\bar{g}^{1 \pm}$ represent the mean transverse acoustic momentum and its deviations on the upper and lower surfaces of the layer, respectively.

The acoustic field in domain $\Omega_{G}$ is obtained as the limit of $P^{\delta}(x)=P^{0, \delta}(x)+\delta P^{1, \delta}(x)+\ldots$ for $\delta \rightarrow 0$ the out-of-layer problem (2) with conditions (4). It can be proved that for the limit problem governing $P^{0}$ the limit transmission layer is totally transparent, so that there is no influence of the perforated plate. However, the first-order correction $P^{1}$ can be computed; for a given $\varepsilon_{0}>0$, hence $\delta_{0}=\varkappa \varepsilon_{0}$, the total response in $\Omega_{\delta_{0}}^{G}$ is given (approximated) by $P^{\delta_{0}} \approx P^{0}+\delta_{0} P^{1}$. In contrast with $P^{0} \in H^{1}\left(\Omega^{G}\right)$ being continuous on $\Gamma_{0}$ representing the limit transmission layer, $P^{1} \in H^{1}\left(\Omega^{+} \cup \Omega^{-}\right)$is discontinuous on $\Gamma_{0}$. Further we introduce the following subdomains $\Omega_{s}^{+}$and $\Omega_{s}^{-}$, and the off-set planes, $\Gamma_{s}^{+}$and $\Gamma_{s}^{-}$, such that

$$
\begin{aligned}
& \Omega_{s}^{+/-}=\left\{x \in \Omega^{+/-} \mid \operatorname{dist}\left(x, \Gamma_{0}\right)<s\right\}, \\
& \Gamma_{s}^{+/-}=\left\{x \in \partial \Omega_{s}^{+/-} \mid \operatorname{dist}\left(x, \Gamma_{0}\right)=s\right\},
\end{aligned}
$$

where $s>0$ can be small; it determines the width of two layers attached to $\Gamma_{0}$ in which the following jump function $\vartheta^{+/-}$is supported. For any $x \in \Omega_{s}^{+/-}$we define $\xi=\operatorname{dist}\left(x, \Gamma_{0}\right)$ and the projection point $x^{0} \in \Gamma_{0}$ of $x$, such that $x=x_{0} \pm \xi \boldsymbol{n}^{0}$, where $\boldsymbol{n}^{0}$ is the unit normal at $\Gamma_{0}$, outward to $\Omega^{-}$. By $\hat{P}=\left[P^{1}\right]_{-}^{+}$we denote the jump on $\Gamma_{0}$ and define

$$
\begin{aligned}
\vartheta^{ \pm}(x) & = \pm \frac{1}{2} \hat{P}\left(x^{0}\right) \bar{\chi}_{ \pm}(x) \hat{\vartheta}(x), \quad \hat{\vartheta} \in H^{1}(\Omega), \\
\hat{\vartheta}(x) & = \begin{cases}1 & \text { for } x \in \Gamma_{0} \\
0 & \text { for } x \in \Gamma_{+/-}^{s} \\
0 & \text { for } x \in \Omega \backslash\left(\Omega_{s}^{+} \cup \Omega_{s}^{-}\right),\end{cases}
\end{aligned}
$$

where $\bar{\chi}_{ \pm}(x)$ is the characteristic function on $\Omega_{s}^{+/-}$, i.e. $\bar{\chi}_{+}(x)=1$ for $x \in \overline{\Omega_{s}^{+}}$and $\bar{\chi}_{+}(x)=0$ for $x \in$ $\Omega \backslash \overline{\Omega_{s}^{+}}$; for $\bar{\chi}_{-}$, the definition is reciprocal. A simplest possible choice is $\hat{\vartheta}(x)=1-\operatorname{dist}\left(x, \Gamma_{0}\right) / s$. Using $\vartheta^{+/-}$, we can express $P^{1}$ in terms of a smooth function $\tilde{P} \in H^{1}(\Omega)$, such that $P^{1}(x)=\tilde{P}(x)+\vartheta^{+/-}(x)$ for $x \in \Omega^{+/-}$.

To compute $P^{1}$, we proceed by evaluating the jumps $\hat{P}$ and $\hat{G}$, the latter representing discontinuity in the first order transverse acoustic momentum. Both these variables depend on $P^{0}$ describing the global acoustic field without any influence of the perforated plate. The following two identities hold,

$$
\begin{array}{r}
\varkappa \int_{\Gamma_{0}} \hat{P} \psi=\int_{\Gamma_{0}} \psi \boldsymbol{D} \cdot \nabla P^{0} \quad \text { for all } \psi \in L^{2}\left(\Gamma_{0}\right), \\
\omega^{2} \int_{\Gamma_{0}} q^{0} \hat{G}=\mathrm{i} \omega \overline{\mathcal{H}}_{0}\left(P^{0}, q^{0}\right)-\omega^{2} c^{2} \int_{\Gamma_{0}} \bar{\nabla}_{x} q^{0} \cdot \boldsymbol{B} \frac{\partial P^{0}}{\partial n}, \quad \text { for all } q^{0} \in H^{1}\left(\Gamma_{0}\right),
\end{array}
$$

where $\boldsymbol{D}=(\boldsymbol{B},-F)$ involves the transverse impedance $F$ depending on the perforation geometry of the plate, and

$$
\overline{\mathcal{H}}_{0}\left(P^{0}, q^{0}\right)=c^{2} \int_{\Gamma_{0}}\left(\boldsymbol{A} \bar{\nabla}_{x} P^{0}\right) \cdot \bar{\nabla}_{x} q^{0}-\omega^{2} \int_{\Gamma_{0}} P^{0} q^{0}
$$

The couple $(\hat{P}, \hat{G})$ is involved in the problem for $\tilde{P} \in H^{1}(\Omega)$ satisfying

$$
\mathcal{H}^{G}(\tilde{P}, Q)=\mathrm{i} \omega \int_{\Gamma_{0}} \hat{G} Q-\mathcal{H}^{+}\left(\vartheta^{+}, Q\right)-\mathcal{H}^{-}\left(\vartheta^{-}, Q\right) \quad \forall Q \in H^{1}(\Omega),
$$

where $\left(\square\right.$ is to be replaced by plus ${ }^{+}$, or minus ${ }^{-}$),

$$
\mathcal{H}^{\square}(P, Q)=c^{2} \int_{\Omega^{\square}} \nabla P \cdot \nabla Q-\omega^{2} \int_{\Omega^{\square}} P Q+\operatorname{ri} \omega c \int_{\partial \Omega^{\square} \cap \partial \Omega^{G}} P Q .
$$



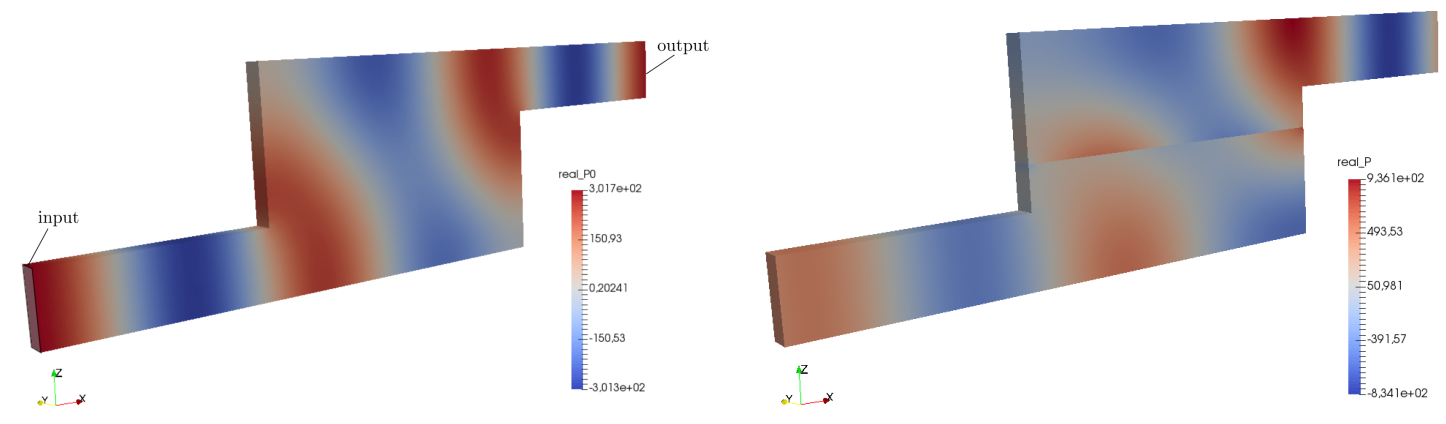

Fig. 2: Distribution of the acoustic potential in a duct: Left: $P^{0}$, no effect of the perforated plate. Right: Total acoustic potential $P^{0}+\delta_{0} P^{1}$ influenced by the perforated interface.

\section{Numerical illustration}

The homogenized model of the transmission layer yield the two interface variables $(\hat{P}, \hat{G})$ as the solution of (8) which has been implemented by the finite element method. To obtain coefficients $\boldsymbol{A}, \boldsymbol{B}$ and $F$, we considered a plate of thickness $h_{0}=1.5 \mathrm{~mm}$ perforated by cylindrical holes with diameter $d_{0}=1.5 \mathrm{~mm}$, which correspond to the scale parameter $\varepsilon_{0}=0.0125$. For illustration of the proposed modelling approach based on the homogenized interface model, we consider a duct in which the perforated plate is fitted. We consider a $2 \mathrm{D}$ problem, such that periodic boundary conditions are prescribed on faces limiting this slice represented as a 3D structure in Fig. 2. The global acoustic problem possessing $P^{0}$ was solved for a plane wave at the input, whereas non-reflection conditions were considered at the output. In Fig. 2, the global acoustic filed $P^{\delta_{0}}$ is displayed as well as the "background" field $P^{0}$ which is independent of the perforated interface.

\section{Conclusions}

We presented a homogenized model of the acoustic transmission on a perforated plate, as an alternative to existing works Rohan and Lukeš (2010), cf. Marigo and Maurel (2016). The global response is constituted by the solution $P^{0}$ of the acoustic problem disregarding the interface and by $\delta_{0}$ multiple of the perturbation $P^{1}$ computed using the gradient $\nabla P^{0}$ evaluated at the interface $\Gamma_{0}$. The proposed modelling approach is based on the rigorous asymptotic analysis of the acoustic field in the layer and the conditions related to the fictitious interfaces between the layer and the outer domains. Extensions for the vibroacoustic problems with deformable plates will be considered in the future, cf. Rohan and Lukeš (2013).

\section{Acknowledgments}

The research and the participation in the conference was supported in part by the grant project GACR 17-01618S and by the the project LO 1506 of the Czech Ministry of Education, Youth and Sports.

\section{References}

Bonnet-Bendhia, A., Drissi, D., Gmati, N. (2005), Mathematical analysis of the acoustic diffraction by a muffler containing perforated ducts. Math. Models and Methods in Appl. Sci., Vol 15, No.7, pp 1059-1090.

Marigo, J.-J., Maurel, A. (2016), Homogenization models for thin rigid structured surfaces and films. J. Acoust. Soc. Am., Vol 140, No.1, pp. 260-273.

Rohan, E., Lukeš, V. (2010), Homogenization of the acoustic transmission through perforated layer, J. of Comput. and Appl. Math., Vol 234, pp. 1876-1885.

Rohan, E., Lukeš, V. (2013), Homogenized perforated interface in acoustic wave propagation - modeling and optimization. In: Proc. of the 11th International Conference on Vibration Problems, ICOVP 2013 (Z. Dimitrovova et.al. Eds.), Lisbon, Portugal, 2013, pp. 1-10, 